\title{
Experienced versus Inexperienced Interexaminer Reliability on Location and Classification of Myofascial Trigger Point Palpation to Diagnose Lateral Epicondylalgia: An Observational Cross-Sectional Study
}

\author{
Raquel Mora-Relucio, ${ }^{1}$ Susana Núñez-Nagy, ${ }^{2}$ Tomás Gallego-Izquierdo, ${ }^{2}$ Alma Rus, ${ }^{3}$ \\ Gustavo Plaza-Manzano, ${ }^{4}$ Natalia Romero-Franco, ${ }^{5}$ \\ Alejandro Ferragut-Garcías, ${ }^{5}$ and Daniel Pecos-Martín ${ }^{2}$ \\ ${ }^{1}$ Free Professional Practice, 28031 Madrid, Spain \\ ${ }^{2}$ Physical Therapy Department, University of Alcalá, Alcalá de Henares, 28806 Madrid, Spain \\ ${ }^{3}$ Cellular Biology Department, University of Granada, 18010 Granada, Spain \\ ${ }^{4}$ Rehabilitation and Physical Medicine Department, Medical Hydrology, Complutense University of Madrid, 28040 Madrid, Spain \\ ${ }^{5}$ Department of Nursing and Physiotherapy, University of the Balearic Islands, 07122 Palma de Mallorca, Spain
}

Correspondence should be addressed to Daniel Pecos-Martín; daniel.pecos@uah.es

Received 10 September 2015; Revised 14 December 2015; Accepted 16 December 2015

Academic Editor: Antonella Fioravanti

Copyright ( 2016 Raquel Mora-Relucio et al. This is an open access article distributed under the Creative Commons Attribution License, which permits unrestricted use, distribution, and reproduction in any medium, provided the original work is properly cited.

\begin{abstract}
The purpose was to evaluate the interexaminer reliability of experienced and inexperienced examiners on location and classification of myofascial trigger points (MTrPs) in two epicondylar muscles and the association between the MTrP found and the diagnosis of lateral epicondylalgia (LE). Fifty-two pianists (some suffered LE) voluntarily participated in the study. Three physiotherapists (one inexperienced in myofascial pain) examined, located, and marked MTrPs in the extensor carpi radialis brevis (ECRB) and extensor digitorum communis (EDC) muscles. Forearms were photographed and analyzed to establish the degree of agreement on MTrPs diagnosis. Data showed $81.73 \%$ and $77.88 \%$ of agreement on $\mathrm{MTrP}$ classification and $85.58 \%$ and $72.12 \%$ on $\mathrm{MTrP}$ location between the expert evaluators for ECRB and EDC, respectively. The agreement on MTrP classification between experienced and inexperienced examiners was $54.81 \%$ and $51.92 \%$ for ECRB and $50.00 \%$ and $55.77 \%$ for EDC. Also, agreement on MTrP location was $54.81 \%$ and $60.58 \%$ for ECRB and $48.08 \%$ and $48.08 \%$ for EDC. A strong association was found between presence of relevant MTrPs, LE diagnosis, and forearm pain when the examiners were experts. The analysis of location and classification of MTrPs in the epicondylar muscles through physical examination by experienced evaluators is reliable, reproducible, and suitable for diagnosing LE.
\end{abstract}

\section{Introduction}

Lateral epicondylalgia (LE), or Tennis Elbow, is characterized by pain over or near the lateral humeral epicondyle or in the forearm extensor muscles and is associated with loss of functional activity. This condition is accompanied by tenderness upon muscle palpation, which may radiate to the forearm and increase with muscle activity $[1,2]$. Several risk factors for
LE development in the working population include repetitive movements of elbow and wrist for more than $2 \mathrm{~h}$ /day [3] due to the overuse of the forearm extensor musculature, especially the extensor carpi radialis brevis (ECRB) [4]. In this regard, professional musicians are a sensible group, given the strict training program to which they are subjected to improve their playing skills [5], with pianists being more particularly prone to suffering from musculoskeletal disorders such as LE $[6,7]$. 
Currently, several reliable techniques are used to diagnose LE, such as elastography [8] and ultrasound [9], but they imply high costs. Therefore, the patient's symptoms are the main criteria normally used to diagnose LE. In this regard, pain evoked by palpation of the myofascial trigger points (MTrPs) in the epicondyle musculature is a key criterion for LE diagnosis.

The MTrP is a hyperirritable focal point within some tense fibers of skeletal muscle. This point is painful under compression and may cause characteristic referred pain, motor dysfunction, and autonomic phenomena [10]. A MTrP may alter muscular contraction patterns and the effectiveness of joint movements [11]. Clinically, MTrPs can be relevant (symptom-producing) or latent (not spontaneously symptomatic) $[12,13]$. Relevant MTrPs evoke the referred pain described by LE patients. Although latent MTrPs are not an immediate source of pain, they may change muscle activation patterns and generate nociceptive behaviors [11]. Moreover, muscle overuse may increase the irritability of latent MTrPs $[10,11]$. MTrP detection is used for the diagnosis of some musculoskeletal pain syndromes $[14,15]$. In some cases, such as fibromyalgia, the presence of MTrPs is one of the main diagnostic criteria [16]. It has been demonstrated that referred pain patterns evoked by compression of MTrPs in the forearm extensor muscles reproduce the pain patterns of LE patients [10], making it useful for LE diagnosis. In this sense, the correct location of MTrPs seems to be essential for diagnosis and subsequent treatment of this pathology [1].

In clinical practice, palpation of MTrPs is affected by the manual skills of examiners. Although a standardized protocol for MTrPs palpation has been proposed by Simons et al. [10], a variety of subjective criteria may influence examiners' diagnosis. Therefore, interexaminer reliability on MTrPs palpation is required to accept it as a valid tool for LE diagnosis [17]. Since the use of innovative technologies to quantify trigger point characteristics and establish a diagnostic criterion standard test is encouraging [18] would be important to prove that the TPs palpation is a reliable and clinically relevant procedure for diagnostic and treatment of the LE.

In this regard, the purpose of this study is to evaluate the interexaminer reliability of experienced and inexperienced examiners on MTrP location and classification in the epicondyle musculature using scanned images. Moreover, associations among examiners' findings, MTrP found and LE diagnosis, and subjects' symptoms were investigated to validate MTrP manual palpation as a diagnostic tool. To the best of our knowledge, this is the first study that analyzes reliability on MTrPs palpation using the novel and complex method of image analysis. The inclusion of inexperienced and experienced examiners will be useful to determine or not the clinical experience as a prerequisite for a valid and reliable LE diagnose.

\section{Methods}

This is an observational cross-sectional study that took place at the University Hospital of the city, during October of 2014. The present study was guided by the STROBE guidelines (see Additional file in Supplementary Material available online at http://dx.doi.org/10.1155/2016/6059719).

2.1. Evaluators. To reduce error chance [19], three physical therapists performed the MTrP evaluation. Two were expert physiotherapists with over 10 years' experience in the diagnosis and treatment of MTrPs and the third was a physical therapist without clinical experience in myofascial pain. All examiners were blinded to LE medical diagnosis of patients.

Prior to the study, two training sessions of one hour each were performed to establish a consensus for MTrPs diagnostic criteria and to practice the procedure to be carried out. These sessions were performed to determine the time required for MTrPs assessment in each subject, as well as the most appropriate area for palpation of MTrPs. The diagnostic criteria included the following: (I) presence of a palpable taut band in a skeletal muscle; (II) presence of a hypersensitive tender spot within the taut band; (III) local twitch response elicited by a snapping palpation of the taut band; and (IV) reproduction of pain in response to MTrP compression. The MTrP was considered relevant if the pain evoked by its compression reproduced the same pain pattern in the patient's elbow and/or forearm. A MTrP was considered nonrelevant if the evoked referred pain did not reproduce a recognizable pain [1].

2.2. Subjects. Fifty-two subjects who were piano students or teachers were randomly recruited from the Musical Education Center "Federico Moreno Torroba" in Madrid (Spain) during September of 2014. All subjects participated voluntarily and signed an informed consent form (the form was signed by the legal representative in case of volunteers under 18 years of age). The following inclusion criteria were established: all subjects were piano students or had completed their studies but continued playing the piano. Those suffering from LE provided the medical diagnosis. The following exclusion criteria were established: shoulder pathology, cervical radiculopathy or whiplash trauma, systemic diseases, cutaneous or subcutaneous alterations in the examination area, determined by visual or palpatory evaluation, and a history of diseases in the upper extremities (previous neck pain, previous neurological disorders, or fractures in the wrist), as well as participants diagnosed with LE, prior to any surgical operation or injection of corticoids because of the antiinflammatory effects of these injections that are believed to relieve pain and diminish disability [20].

The software "Tamaño de la muestra" version 1.1 was employed to calculate the sample size according to Cicchetti $[21,22]$, who reported that sample size calculation in concordance studies using the Kappa coefficient "sample size $=2 k^{2}$ " ( $k=$ categories used in the study) is required. Since three categories were considered ( $0=$ absent MTrPs; 1 = nonrelevant MTrPs; 2 = relevant MTrPs), the sample size required is 36 participants. After adjusting the sample size according to the percentage of losses, 45 participants were needed.

2.3. Materials. Three different-colored markers for topical skin use (AINBOW, CH6004 model) were used to mark MTrPs, marking the skin with invisible and indelible ink. 


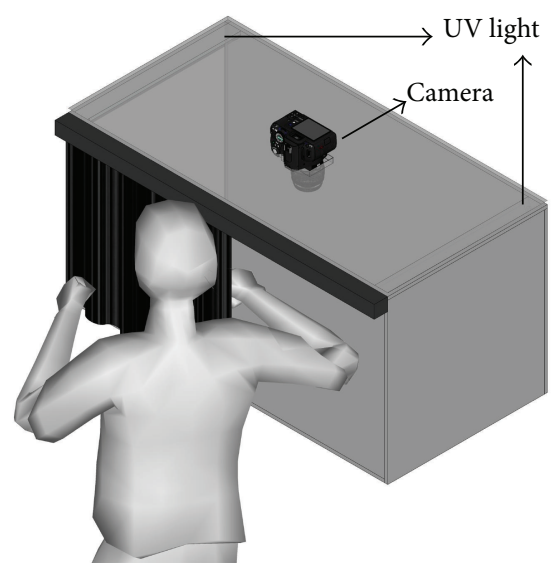

Figure 1: Camera obscura, UV lamps, and digital camera used to obtain images of the myofascial trigger points (MTrPs) marks made by the examiners.

Each examiner used the same marker throughout the procedure. An SLR Digital Camera (Panasonic Lumix DMCG10) was used to photograph the forearms. Two black luminescence tubes (Philips Lighting T8 $18 \mathrm{~W}$ ) were used as the UV light source ( 315 to $400 \mathrm{~nm}$ ). The UV lamps were placed inside a rigid and opaque camera obscura (Figure 1). The digital camera was located over the camera obscura with the lens inward to attenuate ambient light and obtain sharp images of the MTrP marks. Three massage tables were used and the materials were not exchanged or replaced throughout the study to avoid errors during data collection.

2.4. MTrP Evaluation and Myofascial Pain Syndrome Diagnosis Procedure. The experimental procedure was approved by the Ethics Committee of the University Hospital (Ref. OE 29/2011).

The tested muscles were the extensor carpi radialis brevis (ECRB) and the extensor digitorum communis (EDC), given that both are easy to access and were often sore and overused in the pianists.

Each examiner and subject were given a number to preserve confidentiality. At the beginning of the session, an assessor explained the procedure to the participants and collected the medical diagnoses from those subjects with LE. This assessor did not participate in the evaluation process.

Each physical therapist examined the ECRB and EDC muscles of both forearms in all patients to determine the presence of MTrPs. Patients were in a sitting position and had the upper limbs at a $50^{\circ}$ shoulder flexion, elbows propped on the table, the shoulder in neutral rotation, and the forearms in pronation. This procedure was always performed in the same room and under the same ambient conditions.

During the examination, the evaluators were allowed to ask the patients about presence of local or referred pain, pain intensity, and pain recurrence. No more questions were allowed. Although patients were encouraged to interact with examiners, they were not allowed to reveal the previous examiner's findings or condition of LE.
If an examiner found the MTrP in a muscle, the MTrP was classified as relevant or nonrelevant. For this, the MTrP was marked with an invisible ink marker by writing an " $\mathrm{X}$ " for a relevant MTrP or a dot for a nonrelevant MTrP. The use of invisible ink markers ensured that evaluators were blinded to the previous examiner's diagnosis.

Once the examination of the muscles of both forearms was completed by one of the physical therapists, the patient waited alone in the room until the next examiner came. In this way, interferences among examiners could be avoided. This waiting time of 5 min was useful in order to minimize the evidence of hypersensitivity or erythema on the examined area and to avoid a possible examiner bias. The waiting time was calculated by the assessor based on previously published data [23], as well as on the initial training sessions.

2.5. Picture Acquisition and Analysis. Once the procedure was completed, pictures of each patient's forearm were taken in the camera obscura. All pictures were taken by the same photographer using the same photography parameters. To take the picture, the patients adopted the same position as for the assessment. The camera was located $50 \mathrm{~cm}$ above the forearm, and an overhead shot was taken. Each picture included patient number and a scale bar. The UV light revealed the MTrP marks performed by the examiners.

The photographs were reviewed by a different specialist, blinded to the data. The image analysis software AutoCAD 2012 was used to determine the distance between the MTrP marks made by the examiners. The midpoint between the marked MTrPs was first calculated. Using the scale bar contained in each picture as a reference, the distance between the MTrP mark made by Evaluator 1 (E.1) and by Evaluator 2 (E.2) on each muscle was determined, as well as the distance between the E. 2 mark and the one made by Evaluator 3 (E.3). Lastly, the distance between the E.3 and E.1 mark was measured (Figure 2).

2.6. Variables Analyzed. Some categorical variables were created to analyze the agreement between evaluators:

"MTrP classification" is a variable with three categories: Relevant MTrP, Nonrelevant MTrP, or Absent $\mathrm{MTrP}$.

"MTrP location" is a generated variable with two modalities: Agreement and Disagreement. Agreement means that the marks made by the two evaluators are placed within $1.5 \mathrm{~cm}$ of distance. This distance corresponds to the average width of a fingertip, according to the study of Dandekar et al. [24].

"Complete Agreement" is a variable generated to express total agreement on MTrP location and classification. This variable has two levels: Complete agreement and Incomplete agreement. Incomplete agreement refers to a conflict between evaluators about location and/or classification of MTrPs.

2.7. Statistical Analysis. Management and data analysis were performed using the statistical package SPSS for Windows version 19.0 (SPSS Inc., Chicago, IL, USA). All statistical 


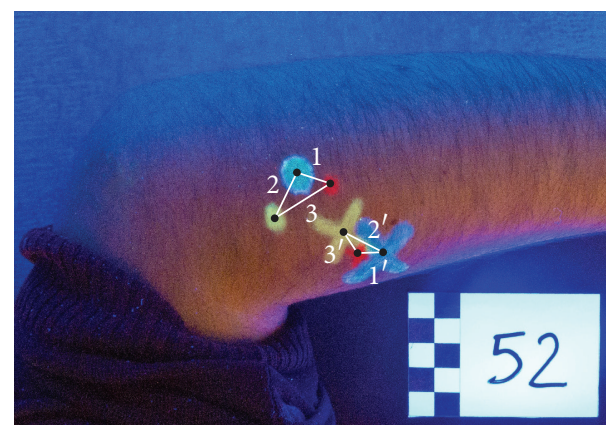

Figure 2: Image of the marks made by the examiners on the epicondyle muscles of each participant.

tests were calculated with a confidence interval of 95\%. Each subject was considered separately.

E.1 evaluations were compared to those of E.2, and those of both experts were compared to the evaluations of E.3.

Pondered Cohen's Kappa Coefficient (quadratic weighting) test for interexaminer agreement was used to determine the agreement on MTrP classification in the ECRB and EDC muscles. This test is a descriptive statistic that measures the agreement between examiners [25]. Quadratic weighting was used because MTrP was classified into three categories $(0=$ $\mathrm{MTrP}$ does not exist; $1=\mathrm{MTrP}$ nonrelevant; $2=\mathrm{MTrP}$ relevant) and this test allows a higher ponderation about the disagreements [25], as it was important to determine the agreement between the three categories of the classification during the MTrPs location.

Cohen's Kappa Coefficient was used to establish interexaminer agreement on MTrP location and the "Complete Agreement" variable. Interexaminer agreement on MTrP location was perceived as a distance $\leq 1.5 \mathrm{~cm}$. If the distance was $>1.5 \mathrm{~cm}$, diagnosis was considered to be distinct. The $95 \%$ confidence interval was included.

The number of agreements on MTrP classification, location, and "Complete Agreement" was also expressed as a percentage (number of agreements/total cases).

Cramer's V Coefficient was used to assess associations between presence of relevant MTrPs in the ECRB and/or EDC and the patient-specific variables "presence of pain in forearms" and "LE medical diagnosis."

The degree of agreement was determined following the criteria proposed by Landis and Koch [26].

\section{Results}

3.1. Demographic Data. A total of 56 volunteers were interviewed for the study. Four did not meet the inclusion criteria and were excluded: two subjects had a history of wrist fracture, one subject had skin alterations on the forearm, and the other suffered from LE and had been treated with corticoids. Therefore, 52 subjects were included in the study, ranging from 8 to 61 years old (mean: 20.77, SD: 12.190). Table 1 shows the characteristics of the study population.
TABLE 1: Demographic and clinical characteristics of subjects $(n=$ $52)$.

\begin{tabular}{lccc}
\hline Variable & Category & Frequency & Percentage \\
\hline \multirow{2}{*}{ Gender } & Male & 36 & $34.62 \%$ \\
& Female & 68 & $65.38 \%$ \\
\hline \multirow{2}{*}{ Piano level } & Basic & 30 & $28.85 \%$ \\
& Professional & 50 & $48.08 \%$ \\
& Teacher & 24 & $23.08 \%$ \\
\hline \multirow{2}{*}{ Forearm pain } & No & 82 & $78.85 \%$ \\
& Yes & 22 & $21.15 \%$ \\
Lateral epicondylalgia & No & 91 & $87.50 \%$ \\
& Yes & 13 & $12.50 \%$ \\
\hline \multirow{2}{*}{ Dominant hand } & Right-handed & 48 & $92.31 \%$ \\
& Left-handed & 3 & $5.77 \%$ \\
& Ambidextrous & 1 & $1.92 \%$ \\
\hline
\end{tabular}

3.2. Interexaminer Reliability on MTrP Classification. Table 2 summarizes the results of the interexaminer reliability on MTrP classification in ECRB and EDC muscles among expert (E.1, E.2) and inexperienced (E.3) examiners. These data suggested a moderate to substantial agreement on MTrP classification in ECRB muscle between experienced observers (Kappa E.1-E.2 = 0.61, 81.73\% of agreement). The agreement was lower when comparing experienced and inexperienced examiners (Kappa E.1-E.3 = 0.39, 54.81\% of agreement; Kappa E.2-E.3 = 0.36, 51.92\% of agreement). The interexaminer reliability on MTrP classification in the EDC muscle between experienced examiners showed a moderate agreement (Kappa E.1-E.2 $=0.52,77.88 \%$ of agreement). However, the agreement between experienced examiners and inexperienced evaluator decreased considerably (Kappa E.1E. $3=0.29,50.00 \%$ of agreement; Kappa E.2-E. $3=0.30,55.77 \%$ of agreement).

3.3. Interexaminer Reliability on MTrP Location. Table 2 shows the results of interexaminer reliability on MTrP location. Data revealed a substantial agreement on MTrP location in the ECRB muscle between experienced examiners (Kappa E.1-E. $2=0.61,85.58 \%$ of agreement). A fair concordance between the experienced evaluators was detected in the EDC (Kappa E.1-E. $2=0.59,72.12 \%$ of agreement). Conversely, the reliability on MTrP location between experienced and inexperienced evaluators decreased to little accordance or absence of concordance in the ECRB (Kappa E.1-E.3 = 0.02, $54.81 \%$ of agreement; Kappa E.2-E.3 $=-0.00,60.58 \%$ of agreement) and EDC muscles (Kappa E.1-E.3 = 0.1941, 48.08\% of agreement; Kappa E.2-E.3 = 0.20, 48.08\% of agreement).

3.4. Interexaminer Reliability on "Complete Agreement". The variable "Complete Agreement" showed different results depending on the experience of the examiners (Table 2). A moderate agreement on both MTrP classification and location in the muscles analyzed was detected between experienced evaluators (Kappa E.1-E.2 $=0.56,70.19 \%$ of 
TABLE 2: Interexaminer reliability on MTrP diagnosis.

\begin{tabular}{|c|c|c|c|c|c|c|}
\hline \multicolumn{7}{|c|}{ Agreement on MTrP classification } \\
\hline \multirow{2}{*}{ Evaluators } & \multicolumn{3}{|c|}{ Extensor carpi radialis brevis } & \multicolumn{3}{|c|}{ Extensor digitorum communis } \\
\hline & Frequency & Percentage & Pondered Kappa Coefficient & Frequency & Percentage & Pondered Kappa Coefficient \\
\hline E.1-E.2 & 85 & $81.73 \%$ & $0.614^{*}$ & 81 & $77.88 \%$ & $0.523^{*}$ \\
\hline E.1-E.3 & 57 & $54.81 \%$ & $0.386^{*}$ & 52 & $50.00 \%$ & $0.289^{*}$ \\
\hline E.2-E.3 & 54 & $51.92 \%$ & $0.3618^{*}$ & 58 & $55.77 \%$ & $0.303^{*}$ \\
\hline \multicolumn{7}{|c|}{ Agreement on MTrP location } \\
\hline \multirow{2}{*}{ Evaluators } & \multicolumn{3}{|c|}{ Extensor carpi radialis brevis } & \multicolumn{3}{|c|}{ Extensor digitorum communis } \\
\hline & Frequency & Percentage & Kappa Coefficient & Frequency & Percentage & Kappa Coefficient \\
\hline E.1-E.2 & 89 & $85.58 \%$ & $0.617^{*}$ & 75 & $72.12 \%$ & $0.593^{*}$ \\
\hline E.1-E.3 & 57 & $54.81 \%$ & $0.016^{*}$ & 50 & $48.08 \%$ & $0.194^{*}$ \\
\hline E.2-E.3 & 63 & $60.58 \%$ & $-0.003^{*}$ & 50 & $48.08 \%$ & $0.196^{*}$ \\
\hline \multicolumn{7}{|c|}{ Complete agreement on MTrP } \\
\hline \multirow{2}{*}{ Evaluators } & \multicolumn{3}{|c|}{ Extensor carpi radialis brevis } & \multicolumn{3}{|c|}{ Extensor digitorum communis } \\
\hline & Frequency & Percentage & Kappa Coefficient & Frequency & Percentage & Kappa Coefficient \\
\hline E.1-E.2 & 73 & $70.19 \%$ & $0.555^{*}$ & 60 & $57.69 \%$ & $0.438^{*}$ \\
\hline E.1-E.3 & 48 & $46.15 \%$ & $0.455^{*}$ & 41 & $39.42 \%$ & $0.224^{*}$ \\
\hline E.2-E.3 & 47 & $45.19 \%$ & $0.297^{*}$ & 44 & $42.31 \%$ & $0.124^{*}$ \\
\hline
\end{tabular}

MTrP: myofascial trigger point; E.1 and E.2: expert evaluators on myofascial diagnosis; E.3: inexperienced evaluator on myofascial diagnosis; Frequency: frequency of agreement between evaluators; Percentage: percentage of agreement between evaluators. $\left({ }^{*}\right)=p$ value $<0.001$.

TABLE 3: Correlation analysis between presence of relevant MTrPs, forearm pain level, and LE medical diagnosis.

\begin{tabular}{lcc}
\hline $\begin{array}{l}\text { Presence of } \\
\text { relevant MTrPs } \\
\text { in EDC and/or }\end{array}$ & $\begin{array}{c}\text { LE diagnosis } \\
\text { ECRB }\end{array}$ & $\begin{array}{c}\text { Presence of forearm } \\
\text { pain }\end{array}$ \\
E.1 & $0.541(p>0$ value $)$ & $\begin{array}{c}\text { Cramer's V } \\
\text { Coefficient }(p \text { value })\end{array}$ \\
E.2 & $0.459(p>0.001)$ & $0.684(p>0.001)$ \\
E.3 & $0.022(p=0.873)$ & $0.606(p>0.001)$ \\
\hline
\end{tabular}

TrP: myofascial trigger point; E.1, E.2: expert evaluators on myofascial diagnosis; E.3: evaluator without experience on myofascial diagnosis; ECRB: extensor carpi radialis brevis; EDC: extensor digitorum communis; LE: lateral epicondylalgia; $\left({ }^{*}\right)=p$ Value $<0.001$.

agreement in the ECRB; Kappa E.1-E.2 = 0.44, 57.69\% of agreement in the EDC). Nonetheless, a fair agreement was found between expert and inexpert examiners in the ECRB (Kappa E.1-E.3 = 0.46, 46.15\% of agreement; Kappa E.2E.3 $=0.30,45.19 \%$ of agreement) and EDC (Kappa E.1-E.3 = $0.22,39.42 \%$ of agreement; Kappa E.2-E.3 $=0.12,42.31 \%$ of agreement).

3.5. Association Analysis. Table 3 shows that the detection of relevant MTrPs in the ECRB and/or EDC by experienced examiners significantly correlated with forearm pain (E.1 = $0.54, p<0.001$; E. $2=0.45, p<0.001)$ and LE medical diagnosis $(\mathrm{E} .1=0.68, p<0.001$; E. $2=0.60, p<0.001)$. Contrarily, the presence of relevant MTrPs, determined by the inexperienced examiner, did not correlate with LE diagnosis (E.3 $=0.02, p=0.873)$, nor the presence of forearm pain $(\mathrm{E} .3=0.123, p=0.400)$.

\section{Discussion}

The purpose of the present study was to investigate the interexaminer reliability on location and classification of MTrP in two epicondylar muscles: the extensor carpi radialis brevis and the extensor digitorum communis muscles, which are commonly implicated $[27,28]$. The study used a previously applied assessment procedure [29], as well as a novel method of image analysis to evaluate the interexaminer reliability of accurate MTrPs location and classification in the epicondyle musculature. Associations among examiners' findings, LE diagnosis, and subjects' symptoms were also investigated.

Findings suggested an acceptable agreement on MTrPs classification in both ECRB and EDC muscles between the expert examiners. Nevertheless, when comparing expert and nonexpert evaluators, the agreement level decreased dramatically. The highest agreement level found on MTrPs classification corresponded to Pondered Cohen's Kappa Coefficient values of 0.617 and 0.593 between experienced evaluators in the ECRB and EDC, respectively, which seems to be appropriate to consider when diagnosing [29]. This level of agreement was similar to that found by Myburgh et al. in patients with neck/shoulder pain [29].

To the best of our knowledge, only two studies have investigated the agreement on MTrPs location between examiners, showing different results $[23,30]$. In one of the studies [23], the agreement did not exceed $21 \%$, while the other [30] demonstrated a good clinical precision amongst experienced clinicians with a degree of concordance between $83 \%$ and $92 \%$. These two studies were conducted in healthy subjects and evaluated the precision of manual palpation when locating nonrelevant MTrPs in the trapezius muscle by 
using a different methodology to the one used in the present study. These are the main problems meaning it is not possible to compare effectively the results of these works with the present study.

This study is the first to examine the interrater reliability location of relevant MTrPs by using photogrammetry measurement techniques to quantify the precision of such a location. The present study has shown an agreement on MTrP location, at a distance $<1.5 \mathrm{~cm}[24,31]$, among expert examiners, of $85.58 \%$ in the case of the ECRB and $72.12 \%$ for the EDC muscle. A distance of $1.5 \mathrm{~cm}$ was considered because of the standard size of the fingertip pulp reported by previous studies [20].

Nevertheless, when comparing MTrPs location between expert evaluators and the inexpert examiner, a strong decrease in the percentage of agreement was found, suggesting that an evaluator's manual skills may play a key role in diagnostic accuracy.

These data also showed that the percentage of accordance on "Complete Agreement" (MTrPs classification and location) decreased among the experts as well as when comparing expert and inexperienced examiners in the muscles analyzed with data concerning MTrPs classification and MTrPs location separately. However, the agreement on "Complete Agreement" among experts remained significantly higher. According to former studies [29, 32-34], our results corroborate that specific training and clinical experience are important keys for MTrPs diagnosis and, thus, to design a correct treatment protocol for patients.

Finally, the association between the presence of MTrPs and patient-specific variables was investigated. Data showed that detection of relevant MTrPs by experienced evaluators correlated with LE diagnosis and presence of forearm pain, suggesting that it is likely to reach an accurate diagnosis of LE by manual palpation of MTrPs without using complex and expensive medical techniques. Curiously, no associations were found in the case of the inexperienced examiner. These findings suggest that the use of algometry to diagnose LE may not be necessary to detect a reduction in pain thresholds, as some investigators have described [35]. In the same way, the use of other techniques, such as Doppler ultrasound [36], elastography [37], or magnetic resonance imaging (MRI), may not be indispensable as diagnostic tools for LE [38]. The present work supports the idea previously proposed [39] that palpation of MTrPs on the epicondyle musculature made by an experienced practitioner is an effective, fast, and inexpensive method to correctly diagnose LE.

This study presents some limitations: First, the findings may not be applied to other syndromes or pain conditions, except LE. Second, the number of examiners included in the study is small.

Regarding the validity of findings, these are limited to superficial forearm muscles and may not be generalized to deeper muscles like the supinator muscle. In addition, despite the acceptable evidence of reproducibility on location and classification of MTrPs, these results should be considered only for expert practitioners. Also, study participants were all pianists, which may hamper the extrapolation of results to the rest of the population. In this regard, further studies considering other populations are needed. Also, further studies may be of interest to compare physical exam results with laboratory findings and imaging tests to support the presence of MTrPs.

These findings show that the diagnosis of MTrPs in the epicondyle musculature through palpation is reliable when the evaluators are expert practitioners. This is supported also by the LE medical diagnosis and forearm pain.

\section{Conclusions}

The use of invisible ink pens and photographic analysis of data have proven to be effective tools to investigate the reliability of LE diagnosis by MTrPs palpation among examiners. The results have shown an acceptable evidence of reproducibility on classification and location of MTrPs in the epicondyle musculature among expert practitioners, while the reliability was significantly lower when comparing with an inexperienced evaluator. Findings also showed that the detection of relevant MTrPs by experienced examiners was associated with LE medical diagnosis and forearm pain, supporting the possibility of achieving a precise LE diagnosis by MTrPs palpation without needing expensive diagnosis techniques. These findings highlight the importance of the experience level of examiners in MTrP palpation for LE diagnosis. Therefore, clinical training of nonexpert physiotherapists may be suggested to improve interexaminer reliability on palpation of MTrPs and LE diagnosis accuracy.

\section{Abbreviations}

MTrP: Myofascial trigger points

LE: Lateral epicondylalgia

EDC: Extensor digitorum communis

ECRB: Extensor carpi radialis brevis.

\section{Conflict of Interests}

The authors declare that they have no competing interests.

\section{Authors' Contribution}

Raquel Mora-Relucio, Daniel Pecos-Martín, Susana NúñezNagy, and Gustavo Plaza-Manzano have made substantial contributions to conception, design, acquisition of data, analysis, and interpretation of data. Tomás Gallego-Izquierdo, Alma Rus, and Natalia Romero-Franco have been involved in drafting the paper or revising it critically for important intellectual content. Natalia Romero-Franco, Tomás Gallego-Izquierdo, Daniel Pecos-Martín, and Gustavo PlazaManzano have given final approval of the version to be published. All the authors agree to be accountable for all aspects of the work in ensuring that questions related to the accuracy or integrity of any part of the work are appropriately investigated and resolved. 


\section{References}

[1] J. Fernández-Carnero, C. Fernández-De-Las-Peñas, A. I. de La Llave-Rincón, H.-Y. Ge, and L. Arendt-Nielsen, "Prevalence of and referred pain from myofascial trigger points in the forearm muscles in patients with lateral epicondylalgia," Clinical Journal of Pain, vol. 23, no. 4, pp. 353-360, 2007.

[2] T. Pienimäki, T. Tarvainen, P. Siira, A. Malmivaara, and H. Vanharanta, "Associations between pain, grip strength, and manual tests in the treatment evaluation of chronic tennis elbow," Clinical Journal of Pain, vol. 18, no. 3, pp. 164-170, 2002.

[3] E. Herquelot, A. Guéguen, Y. Roquelaure et al., "Work-related risk factors for incidence of lateral epicondylitis in a large working population," Scandinavian Journal of Work, Environment and Health, vol. 39, no. 6, pp. 578-588, 2013.

[4] B. O. Ljung, R. L. Lieber, and J. Friden, "Wrist extensor muscle pathology in lateral epicondylitis," Journal of Hand SurgeryBritish Volume, vol. 24, no. 2, pp. 177-183, 1999.

[5] F. J. Bejjani, G. M. Kaye, and M. Benham, "Musculoskeletal and neuromuscular conditions of instrumental musicians," Archives of Physical Medicine and Rehabilitation, vol. 77, no. 4, pp. 406413, 1996.

[6] P. Bragge, A. Bialocerkowski, and J. McMeeken, "A systematic review of prevalence and risk factors associated with playingrelated musculoskeletal disorders in pianists," Occupational Medicine, vol. 56, no. 1, pp. 28-38, 2006.

[7] R. J. Lederman, "Neuromuscular and musculoskeletal problems in instrumental musicians," Muscle and Nerve, vol. 27, no. 5, pp. 549-561, 2003.

[8] Q. Chen, S. Bensamoun, J. R. Basford, J. M. Thompson, and K.-N. An, "Identification and quantification of myofascial taut bands with magnetic resonance elastography," Archives of Physical Medicine and Rehabilitation, vol. 88, no. 12, pp. 1658-1661, 2007.

[9] S. Sikdar, J. P. Shah, T. Gebreab et al., "Novel applications of ultrasound technology to visualize and characterize myofascial trigger points and surrounding soft tissue," Archives of Physical Medicine and Rehabilitation, vol. 90, no. 11, pp. 1829-1838, 2009.

[10] D. G. Simons, J. G. Travell, and L. S. Simons, Travell \& Simons' Myofascial Pain and Dysfunction: Upper Half of Body, Lippincott Williams \& Wilkins, Philadelphia, Pa, USA, 1999.

[11] K. R. Lucas, P. A. Rich, and B. I. Polus, "Muscle activation patterns in the scapular positioning muscles during loaded scapular plane elevation: the effects of latent myofascial trigger points," Clinical Biomechanics, vol. 25, no. 8, pp. 765-770, 2010.

[12] D. G. Simons, "Review of enigmatic MTrPs as a common cause of enigmatic musculoskeletal pain and dysfunction," Journal of Electromyography and Kinesiology, vol. 14, no. 1, pp. 95-107, 2004.

[13] R. D. Gerwin, J. Dommerholt, and J. P. Shah, "An expansion of Simons' integrated hypothesis of trigger point formation," Current Pain and Headache Reports, vol. 8, no. 6, pp. 468-475, 2004.

[14] R. Henry, C. M. Cahill, G. Wood et al., "Myofascial pain in patients waitlisted for total knee arthroplasty," Pain Research and Management, vol. 17, no. 5, pp. 321-327, 2012.

[15] C. Bron, J. Dommerholt, B. Stegenga, M. Wensing, and R. A. Oostendorp, "High prevalence of shoulder girdle muscles with myofascial trigger points in patients with shoulder pain," $B M C$ Musculoskeletal Disorders, vol. 12, article 139, 2011.
[16] F. Wolfe, H. A. Smythe, M. B. Yunus et al., "The American College of Rheumatology 1990 Criteria for the classification of fibromyalgia," Arthritis and Rheumatism, vol. 33, no. 2, pp. 160$172,1990$.

[17] N. Lucas, P. Macaskill, L. Irwig, R. Moran, and N. Bogduk, "Reliability of physical examination for diagnosis of myofascial trigger points: a systematic review of the literature," Clinical Journal of Pain, vol. 25, no. 1, pp. 80-89, 2009.

[18] C. Myburgh, A. H. Larsen, and J. Hartvigsen, "A systematic, critical review of manual palpation for identifying myofascial trigger points: evidence and clinical significance," Archives of Physical Medicine and Rehabilitation, vol. 89, no. 6, pp. 11691176, 2008.

[19] W. Vach, “The dependence of Cohen's kappa on the prevalence does not matter," Journal of Clinical Epidemiology, vol. 58, no. 7, pp. 655-661, 2005.

[20] N. Smidt, W. J. J. Assendelft, D. A. W. M. van der Windt, E. M. Hay, R. Buchbinder, and L. M. Bouter, "Corticosteroid injections for lateral epicondylitis: a systematic review," Pain, vol. 96, no. 1-2, pp. 23-40, 2002.

[21] D. V. Cicchetti, "Testing the normal approximation and minimal sample size requirements of weighted kappa when the number of categories is large," Applied Psychological Measurement, vol. 5, no. 1, pp. 101-104, 1981.

[22] D. V. Cicchetti and J. L. Fleiss, "Comparison of the null distributions of weighted kappa and the C ordinal statistic," Applied Psychological Measurement, vol. 1, no. 2, pp. 195-201, 1977.

[23] P. C. Lew, J. Lewis, and I. Story, "Inter-therapist reliability in locating latent myofascial trigger points using palpation," Manual Therapy, vol. 2, no. 2, pp. 87-90, 1997.

[24] K. Dandekar, B. I. Raju, and M. A. Srinivasan, "3-D finiteelement models of human and monkey fingertips to investigate the mechanics of tactile sense," Journal of Biomechanical Engineering, vol. 125, no. 5, pp. 682-691, 2003.

[25] M. J. Warrens, "Weighted kappa is higher than Cohen's kappa for tridiagonal agreement tables," Statistical Methodology, vol. 8, no. 2, pp. 268-272, 2011.

[26] J. R. Landis and G. G. Koch, "A one-way components of variance model for categorical data," Biometrics, vol. 33, no. 4, pp. 671679, 1977.

[27] F. Faro and J. M. Wolf, "Lateral epicondylitis: review and current concepts," The Journal of Hand Surgery-American Volume, vol. 32, no. 8, pp. 1271-1279, 2007.

[28] S. M. Fairbank and R. J. Corlett, "The role of the extensor digitorum communis muscle in lateral epicondylitis," Journal of Hand Surgery, vol. 27, no. 5, pp. 405-409, 2002.

[29] C. Myburgh, H. H. Lauridsen, A. H. Larsen, and J. Hartvigsen, "Standardized manual palpation of myofascial trigger points in relation to neck/shoulder pain; the influence of clinical experience on inter-examiner reproducibility," Manual Therapy, vol. 16, no. 2, pp. 136-140, 2011.

[30] V. M. Sciotti, V. L. Mittak, L. DiMarco et al., "Clinical precision of myofascial trigger point location in the trapezius muscle," Pain, vol. 93, no. 3, pp. 259-266, 2001.

[31] E. R. Serina, C. D. Mote Jr., and D. Rempel, "Force response of the fingertip pulp to repeated compression-effects of loading rate, loading angle and anthropometry," Journal of Biomechanics, vol. 30, no. 10, pp. 1035-1040, 1997. 
[32] C.-Y. J. Hsieh, C.-Z. Hong, A. H. Adams et al., "Interexaminer reliability of the palpation of trigger points in the trunk and lower limb muscles," Archives of Physical Medicine and Rehabilitation, vol. 81, no. 3, pp. 258-264, 2000.

[33] R. D. Gerwin, S. Shannon, C.-Z. Hong, D. Hubbard, and R. Gevirtz, "Interrater reliability in myofascial trigger point examination," Pain, vol. 69, no. 1-2, pp. 65-73, 1997.

[34] A. M. Al-Shenqiti and J. A. Oldham, "Test-retest reliability of myofascial trigger point detection in patients with rotator cuff tendonitis," Clinical Rehabilitation, vol. 19, no. 5, pp. 482-487, 2005.

[35] J. Fernández-Carnero, C. Fernández-de-las-Peñas, M. Sterling, T. Souvlis, L. Arendt-Nielsen, and B. Vicenzino, "Exploration of the extent of somato-sensory impairment in patients with unilateral lateral epicondylalgia," Journal of Pain, vol. 10, no. 11, pp. 1179-1185, 2009.

[36] C. du Toit, M. Stieler, R. Saunders, L. Bisset, and B. Vicenzino, "Diagnostic accuracy of power Doppler ultrasound in patients with chronic tennis elbow," British Journal of Sports Medicine, vol. 42, no. 11, pp. 872-876, 2008.

[37] T. De Zordo, S. R. Lill, C. Fink et al., "Real-time sonoelastography of lateral epicondylitis: comparison of findings between patients and healthy volunteers," American Journal of Roentgenology, vol. 193, no. 1, pp. 180-185, 2009.

[38] M. Aoki, T. Wada, S. Isogai, K. Kanaya, H. Aiki, and T. Yamashita, "Magnetic resonance imaging findings of refractory tennis elbows and their relationship to surgical treatment," Journal of Shoulder and Elbow Surgery, vol. 14, no. 2, pp. 172177, 2005.

[39] B. S. Kraushaar and R. P. Nirschl, "Tendinosis of the elbow (tennis elbow). Clinical features and findings of histological, immunohistochemical, and electron microscopy studies," The Journal of Bone \& Joint Surgery-American Volume, vol. 81, no. 2, pp. 259-278, 1999. 


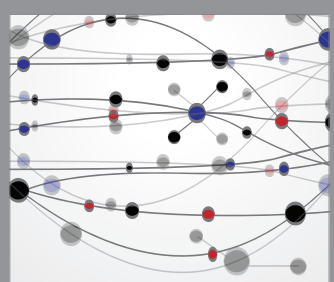

The Scientific World Journal




Gastroenterology Research and Practice

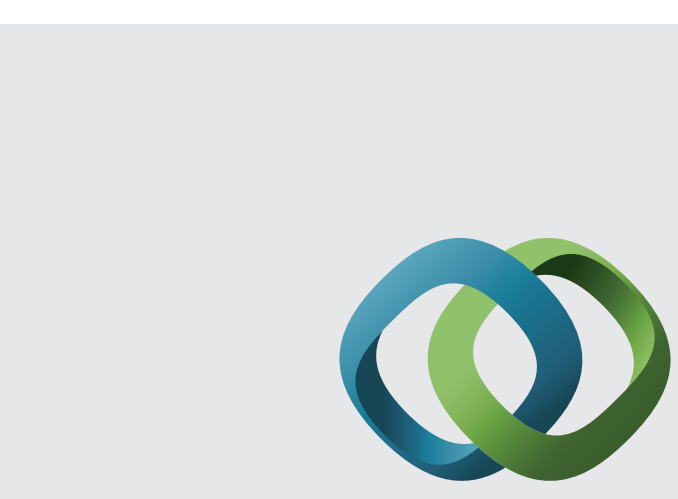

\section{Hindawi}

Submit your manuscripts at

http://www.hindawi.com
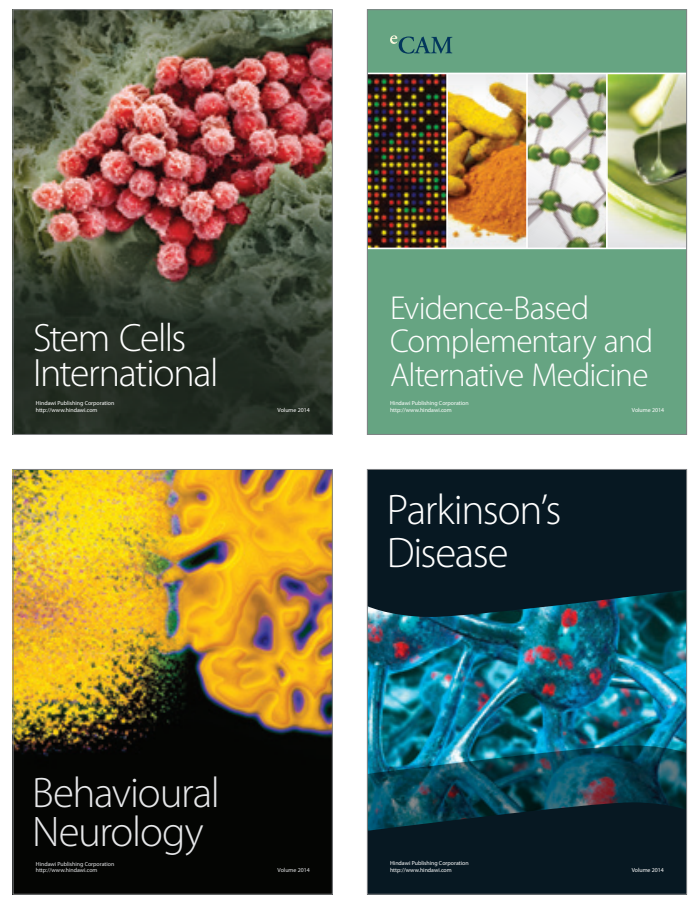
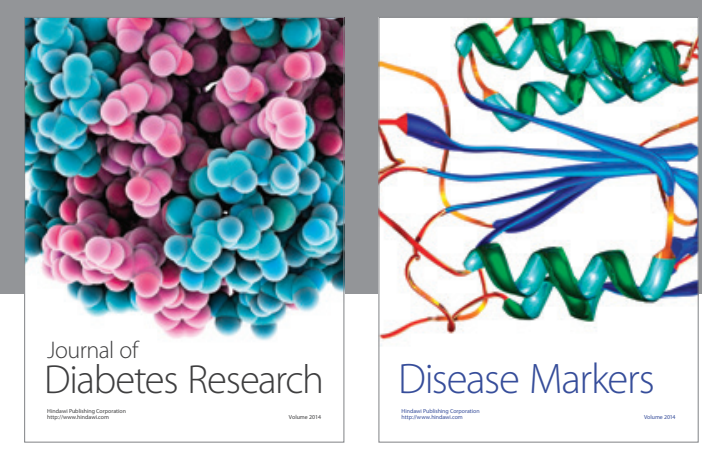

Disease Markers
